From the University Medical Center Groningen, University of Groningen, Groningen; Radboud University Nijmegen Medical Center, Nijmegen; Erasmus Medical Center, Rotterdam; VU University Medical Center; Academic Medical Center, Amsterdam; Leiden University Medical Center, Leiden: University Medical Center Utrecht, Utrecht: Maastricht University Medical Center, Maastricht; Catharina Hospital Eindhoven, the Netherlands; University Hospitals Leuven, Leuven, Belgium; European Cancer Institute, Milan, Italy; Addenbrooke's Hospital, Cambridge, United Kingdom; Hôpital Notre DameCentre Hospitalier de I'Université de Montreal, Montreal, Quebec, Canada; and Charité-Universitätsmedizin Berlin, Berlin, Germany.

Submitted August 20, 2007; accepted October 4, 2007

Presented at the 11th Biennial Meeting of the International Gynecologic Cance Society, October 14-18, 2006, Santa Monica, CA, and the Society of Gynecologic Oncologists 37th Annual Meeting on Women's Cancer, March 22-26, 2006, Palm Springs, CA.

Authors' disclosures of potential conflicts of interest and author contributions are found at the end of this article.

Corresponding author: Ate G.J. Van der Zee, MD, PhD, Department of Obstetrics and Gynecology, University Medical Center Groningen, PO Box 30.001, 9700 RB Groningen, the Netherlands: e-mail: a.g.j.van.der.zee@og.umcg.nl.

(c) 2008 by American Society of Clinical Oncology

0732-183X/08/2606-884/\$20.00

DOI: $10.1200 / J C O .2007 .14 .0566$

\title{
Sentinel Node Dissection Is Safe in the Treatment of Early-Stage Vulvar Cancer
}

Ate G.J. Van der Zee, Maaike H. Oonk, Joanne A. De Hullu, Anca C. Ansink, Ignace Vergote, René H. Verheijen, Angelo Maggioni, Katja N. Gaarenstroom, Peter J. Baldwin, Eleonore B. Van Dorst, Jacobus Van der Velden, Ralph H. Hermans, Hans van der Putten, Pierre Drouin, Achim Schneider, and Wim J. Sluiter

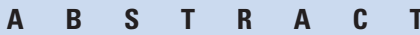

\section{Purpose}

To investigate the safety and clinical utility of the sentinel node procedure in early-stage vulvar cancer patients.

\section{Patients and Methods}

A multicenter observational study on sentinel node detection using radioactive tracer and blue dye was performed in patients with T1/2 $(<4 \mathrm{~cm})$ squamous cell cancer of the vulva. When the sentinel node was found to be negative at pathologic ultrastaging, inguinofemoral lymphadenectomy was omitted, and the patient was observed with follow-up for 2 years at intervals of every 2 months. Stopping rules were defined for the occurrence of groin recurrences.

\section{Results}

From March 2000 until June 2006, a sentinel node procedure was performed in 623 groins of 403 assessable patients. In 259 patients with unifocal vulvar disease and a negative sentinel node (median follow-up time, 35 months), six groin recurrences were diagnosed $(2.3 \% ; 95 \% \mathrm{Cl}, 0.6 \%$ to $5 \%)$, and 3 -year survival rate was $97 \%(95 \% \mathrm{Cl}, 91 \%$ to $99 \%)$. Short-term morbidity was decreased in patients after sentinel node dissection only when compared with patients with a positive sentinel node who underwent inguinofemoral lymphadenectomy (wound breakdown in groin: $11.7 \% \vee 34.0 \%$, respectively; $P<.0001$; and cellulitis: $4.5 \% \vee 21.3 \%$, respectively; $P<.0001)$. Long-term morbidity also was less frequently observed after removal of only the sentinel node compared with sentinel node removal and inguinofemoral lymphadenectomy (recurrent erysipelas: $0.4 \% \vee 16.2 \%$, respectively; $P<.0001$; and lymphedema of the legs: $1.9 \%$ $\checkmark 25.2 \%$, respectively; $P<.0001$ ).

\section{Conclusion}

In early-stage vulvar cancer patients with a negative sentinel node, the groin recurrence rate is low survival is excellent, and treatment-related morbidity is minimal. We suggest that sentinel node dissection, performed by a quality-controlled multidisciplinary team, should be part of the standard treatment in selected patients with early-stage vulvar cancer.

\section{J Clin Oncol 26:884-889. (c) 2008 by American Society of Clinical Oncology}

\section{INTRODUCTION}

Squamous cell cancer of the vulva is a rare disease with an annual incidence of two to three per 100,000 women. $^{1-3}$ Current standard treatment for earlystage disease consists of radical excision of the tumor with elective inguinofemoral lymphadenectomy. The efficacy of this treatment is good, with reported groin recurrence rates varying between $1 \%$ and $10 \%$. $^{4-11}$ However, only $25 \%$ to $35 \%$ of patients with early-stage disease will have lymph node metastases, ${ }^{5-9}$ and the remaining $65 \%$ to $75 \%$ of patients are unlikely to benefit from elective inguinofemoral lymphadenectomy but will be at risk for its significant morbidity. In the short term, wound healing in the groin is compromised by infection and breakdown in $20 \%$ to $40 \%$ of patients. In the long term, lymphedema of the legs with increased risk for erysipelas occurs in 30\% to $70 \%$ of patients. ${ }^{10,12-14}$ Despite significant surgical morbidity and a low frequency of lymph node metastases, an elective lymphadenectomy is regarded as standard of care because unrecognized disease in the inguinofemoral lymph nodes is nearly always fatal. A noninvasive or minimally invasive technique that allows the detection of inguinofemoral metastases with a low false-negative rate is desirable. To date, noninvasive imaging techniques are neither 
sensitive nor specific enough for the detection of (micro)metastases in inguinofemoral lymph nodes. ${ }^{15,16}$

In a variety of malignancies, such as breast cancer and cutaneous melanoma, the false-negative rate of the sentinel node procedure seems to be low (range, $0 \%$ to $29 \%$; average, $7.3 \%$ ). ${ }^{17,18}$ In breast cancer, not only was the nodal recurrence rate in the axilla exceptionally low $(0.1 \%$ to $0.3 \%)$, but the sentinel node procedure was also associated with lower morbidity and improved quality of life when compared with complete lymphadenectomy. ${ }^{19-23}$ Studies in vulvar cancer in which sentinel node detection was followed by inguinofemoral lymphadenectomy suggest that the sentinel node procedure is highly accurate in identifying lymph node metastases with a negative predictive value approaching $100 \% .{ }^{24-34}$ However, safety and clinical utility still need to be proven in large clinical trials. The optimal design would be an equivalence randomized trial in which patients with a negative sentinel node are randomly assigned to either observation alone or inguinofemoral lymphadenectomy. However, because of the low incidence of the disease and the high number of patients needed, several collaborative groups in gynecologic cancer deemed such a design as highly unrealistic.

The aims of this observational study were to investigate the safety of omitting inguinofemoral lymphadenectomy in patients with a negative sentinel node and to compare short- and long-term morbidity between sentinel node removal only and inguinofemoral lymphadenectomy performed in patients with a positive sentinel node. Groin recurrences were regarded as the most serious short-term threat for participating patients with a negative sentinel node. Therefore, for safety reasons, stopping rules were formulated, using continuous sequential analysis of the occurrence of groin recurrences in the first 2 years of follow-up for each patient.

\section{PATIENTS AND METHODS}

\section{Patients}

Eligible patients had $\mathrm{T} 1$ or $\mathrm{T} 2$, less than $4 \mathrm{~cm}$, squamous cell cancer of the vulva with a depth of invasion more than $1 \mathrm{~mm}$ and clinically nonsuspicious inguinofemoral lymph nodes. To ensure the quality of the sentinel node procedure in the participating centers, it was determined that each gynecologic oncology center needed to have documented successful experience of the sentinel node procedure with subsequent inguinofemoral lymphadenectomy in at least 10 vulvar cancer patients (see Appendix, online only). Permission from all local ethics committees was obtained. All patients gave written informed consent. Patients were only included when registered at the University Medical Center Groningen (Groningen, the Netherlands) before the start of treatment of the patient. Central data management was performed at the University Medical Center Groningen.

\section{Sentinel Node Detection and Treatment Protocol}

Treatment consisted of radical excision of the primary tumor in combination with sentinel node procedure. The sentinel node procedure was performed with the combined technique (radioactive tracer and blue dye), as previously described. ${ }^{26}$ After removal, sentinel nodes were sent to the pathologist as individual specimens. When the sentinel node was negative, no further treatment followed. If metastatic disease was identified in the sentinel node, an inguinofemoral lymphadenectomy was performed, either during the same operation when found at frozen sectioning or during a subsequent operation when found at routine pathologic examination or ultrastaging. When more than one intranodal metastasis and/or extranodal growth was detected, postoperative external-beam radiotherapy (50 Gy) to the groin/pelvis was recommended (treatment protocol is detailed in Appendix).

\section{Morbidity and Follow-Up}

Data on postoperative morbidity were collected in a prospective fashion. Short-term morbidity was defined as the occurrence of wound breakdown or wound infection (requiring antibiotics). Patients were seen at least every 2 months for the first 2 years after treatment. Assessment included interview, gynecologic examination, and palpation of the groins. The presence of lymphedema (objective findings and subjective symptoms) and/or erysipelas of the legs was documented. Long-term morbidity, defined by either lymphedema (present at two consecutive follow-up visits $>1$ year after primary therapy) and/or recurrent erysipelas ( $>$ one episode of erysipelas requiring antibiotics), was also documented.

\section{Histopathology}

The pathologic assessment of the sentinel node(s) was performed according to a standard protocol. Briefly, the sentinel nodes were cut in the middle for frozen section or cytologic specimen (both optional). Subsequently, four sections were cut from every half for hematoxylin and eosin (HE) staining (routine histopathologic examination). Ultrastaging was performed only on sentinel nodes that were negative on routine histopathologic examination. For ultrastaging, additional pairs of sections were cut with three sections per millimeter. One section of each pair was stained with $\mathrm{HE}$, and the other section was immunostained with cytokeratin 1\% AE1:AE3 antikeratin solution (Boehringer Mannheim, Mannheim, Germany). From the lymphadenectomy specimens, all lymph nodes were studied individually (one section per $0.5 \mathrm{~cm}$ for HE staining).

\section{Sample Size}

From the literature, the groin recurrence rate after inguinofemoral lymphadenectomy and no evidence of nodal metastases was conservatively estimated to be $2 \%$ in early-stage vulvar cancer. ${ }^{4-11}$ An increase in groin recurrence of $6 \%$ was considered to be the maximum acceptable increase in light of an anticipated significant decrease in treatment-related morbidity. Our power calculation was based on the assumption that all groin recurrences would become evident within the first 2 years of follow-up. To exclude a failure rate of $8 \%$ after 2 years compared with $2 \%, 219$ patients with 2 years of follow-up were needed to reach a two-sided level of significance of $5 \%$ with $90 \%$ power. With final analysis 1 year after the last entry and an accrual rate of approximately 50 patients per year, the total number of patients would be 244 . Censoring of an estimated 30 patients for local recurrence and death not related to vulvar cancer within 2 years would bring the needed number to 259 patients with a negative sentinel node.

\section{Stopping Rules}

To check the efficacy of our treatment protocol, stopping rules were made regarding the rate of groin recurrences in patients with a negative sentinel node (Fig 1). Inferiority of this failure rate to $0.02(\mathrm{H} 0: P=.02 ; \mathrm{H} 1$ : $P=.08$ ) was tested in a fully sequential design. A concomitant test of superiority to 0.08 ( $\mathrm{H} 0: P=.08 ; \mathrm{H} 1: P=.02)$ was used as a test for futility. Both tests used a constant value of $\alpha$, cumulating to .05 with $90 \%$ power. The ethical committee of each participating hospital would be informed of the activation of the stopping rule, and after the analysis of the data, the ethical committees would be requested to judge the proposal of the investigators, based on the results of this analysis, regarding the following: the closure of the study; or the progression of the study with an amended protocol; or the progression of the study with an unchanged protocol (stopping rule design detailed in Appendix).

\section{Statistics}

All analyses were performed using SPSS software, version 11 (SPSS Inc, Chicago, IL). Differences in the distributions of patient characteristics were analyzed with the $\chi^{2}$ test. Differences between characteristics such as tumor diameter were tested with the $t$ test. We performed analyses of survival using the Kaplan-Meier method. Differences associated with a $P<.05$ were considered significant. Follow-up and survival rates were calculated from the date of primary surgery to the date of last examination or death. 


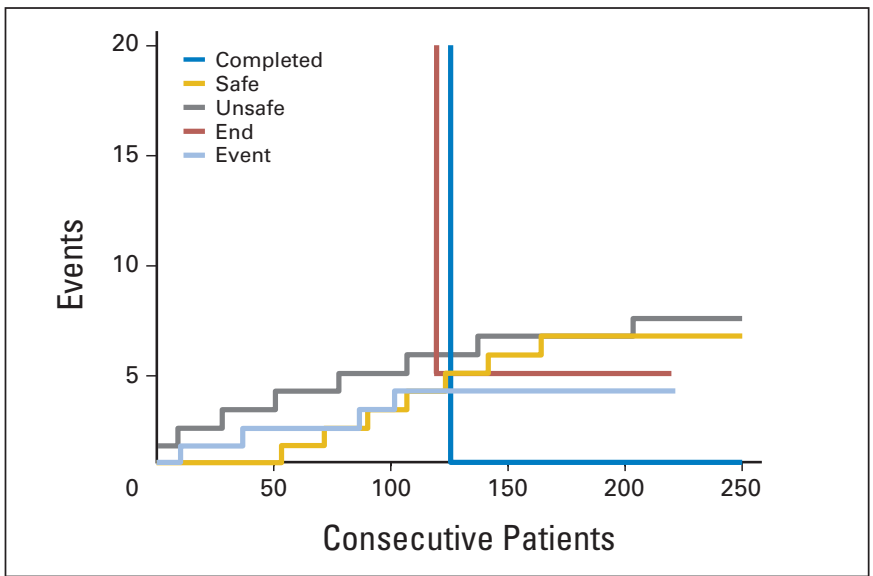

Fig 1. Stopping rules for patients with unifocal disease. The gray line indicates inferiority to a groin recurrence rate of 0.02 ; the yellow line indicates superiority to 0.08 . The yellow ("safe") boundary was passed by the light blue events line when 126 patients with unifocal vulvar cancer and a negative sentinel lymph node had completed 2 years of follow-up without groin recurrences. Patients were censored at time of death by other causes than vulvar cancer or local recurrence.

\section{RESULTS}

\section{Patients}

From March 2000 until June 2006, 457 patients from 15 centers were registered (see Appendix). All centers had fulfilled the quality criteria. The median number of registered patients per center was 21 (range, three to 113 patients). In 403 patients, a sentinel node procedure according to the protocol was performed. The clinical characteristics of these patients are listed in Table 1. In 127 (31.5\%) of 403 patients, pathology showed $\geq$ one metastatic sentinel node, and therefore, a total of $276(68.5 \%)$ of 403 patients were eligible for the observational study.

\section{Sentinel Node Characteristics}

In 623 groins of 403 patients, a sentinel node procedure was performed (183 patients only unilateral). In 163 groins (26.2\%), metastatic sentinel node(s) were found. Routine pathologic examination detected 95 (58.3\%) of 163 groins with metastatic sentinel

Table 1. Characteristics of 403 Patients Who Underwent the Sentinel Node Procedure According to the Protocol

\begin{tabular}{lcr}
\hline \multicolumn{1}{c}{ Characteristic } & No. of Patients & $\%$ \\
\hline Location of primary tumor* & 151 & 37.5 \\
Lateral & 252 & 62.5 \\
Midline & & \\
Disease & 377 & 93.5 \\
Unifocal & 26 & 6.5 \\
Multifocal & & \\
Treatment of vulvar tumor & 358 & 88.8 \\
Wide local excision & 41 & 10.2 \\
Radical vulvectomy & 4 & 1.0 \\
Radiotherapy & 4
\end{tabular}

*A lateral tumor is defined as a tumor that has a median margin located $\geq 1$ $\mathrm{cm}$ from the midline. A midline tumor is defined as a tumor that has a median margin located $<1 \mathrm{~cm}$ from the midline.

tBecause of localization close to the anal sphincter. nodes, and ultrastaging detected $68(41.7 \%)$ of 163 groins with metastatic sentinel nodes.

\section{Short-Term and Long-Term Complications}

For analyses of short-term morbidity, only patients who underwent a sentinel node procedure and inguinofemoral lymphadenectomy within the same procedure were included $(n=47)$. In the observational study, 12 patients underwent inguinofemoral lymphadenectomy in the contralateral groin. For morbidity analyses, they were included in the lymphadenectomy group. Fourteen patients were excluded because they received radiotherapy instead of lymphadenectomy, three patients refused, two patients died, and one patient had recurrent disease before start of adjuvant treatment. Both short-term and long-term morbidity were less common in patients who underwent sentinel node removal alone compared with patients with a metastatic sentinel node who subsequently underwent inguinofemoral lymphadenectomy (Table 2). Patients who received postoperative radiotherapy in addition to lymphadenectomy experienced recurrent erysipelas more frequently than patients who underwent lymphadenectomy without postoperative radiotherapy (5.9\% v 30.6\%, respectively; $P<.0001$ ). The median hospital stay for patients after sentinel node removal only was 8.4 days compared with 13.7 days for patients who underwent an inguinofemoral lymphadenectomy immediately after a sentinel node was found positive at frozen section $(P<.0001)$.

\section{Stopping Rules}

In October 2003, of 139 patients with a negative sentinel node on study, two patients with multifocal disease (two separate vulvar lesions

Table 2. Short- and Long-Term Morbidity After SLN Procedure Alone Compared With SLN With Subsequent Inguinofemoral Lymphadenectomy

\begin{tabular}{|c|c|c|c|}
\hline Morbidity & $\begin{array}{c}\text { SLN } \\
\text { Dissection Only }\end{array}$ & $\begin{array}{c}\text { SLN } \\
\text { Dissection Plus } \\
\text { Lymphadenectomy }\end{array}$ & $P$ \\
\hline \multicolumn{4}{|l|}{ Short term } \\
\hline Total No. of patients & 264 & $47^{*}$ & \\
\hline Wound breakdown, groin & & & $<.0001$ \\
\hline No. of patients & 31 & 16 & \\
\hline$\%$ & 11.7 & 34.0 & \\
\hline Cellulitis & & & $<.0001$ \\
\hline No. of patients & 12 & 10 & \\
\hline$\%$ & 4.5 & 21.3 & \\
\hline Hospital stay, days & 8.4 & 13.7 & $<.0001$ \\
\hline \multicolumn{4}{|l|}{ Long term } \\
\hline Total No. of patients & 264 & $119 \dagger$ & \\
\hline Lymphedema & & & $<.0001$ \\
\hline No. of patients & 5 & 30 & \\
\hline$\%$ & 1.9 & 25.2 & \\
\hline Recurrent erysipelas & & & $<.0001$ \\
\hline No. of patients & 1 & $19 \ddagger$ & \\
\hline$\%$ & 0.4 & 16.2 & \\
\hline
\end{tabular}

Abbreviation: SLN, sentinel lymph node.

*For comparison of short-term morbidity, only patients who had a complete lymphadenectomy within the same procedure as the SLN dissection procedure were included in the analysis ( $n=47$ ).

tLong-term morbidity was compared between 264 patients after SLN dissection only (276 patients in the observational study minus four patients who had unilateral lymphadenectomy because of an SLN dissection that was not found, and minus eight patients who had one positive node in one groin in the observational study and had a lymphadenectomy on the contralateral side) and 119 patients who had undergone full lymphadenectomy either in the same session as the SLN procedures or at a second procedure $(n=119)$.

$\neq$ Out of a total of 117 patients. 
that were injected separately) and a negative sentinel node suffered from a groin recurrence within a short period of time. Despite the fact that the stopping rules had not yet been activated, it was decided to amend the protocol and to further exclude patients with multifocal disease. This amendment was approved by the ethics committees in all participating centers. Figure 1 shows the performance of the stopping rule for the observational study in unifocal patients at the time of activation because of passing the safety border. At that time, the groin recurrence rate for patients who had completed 2 years of follow-up ( $\mathrm{n}$ $=126)$ was $4.0 \%(95 \% \mathrm{CI}, 1.6 \%$ to $8.0 \%)$. After reaching the number of 259 patients with unifocal disease and a negative sentinel node, the study was closed in June 2006.

\section{Follow-Up in Sentinel Node-Negative Patients}

Groin recurrences. The median follow-up time of the 276 patients in the observational study (17 with multifocal disease) was 35 months (range, 2 to 87 months; date of final analysis: July 1, 2007). Four patients without recurrence were lost to follow-up and did not complete the minimum of 24 months of follow-up. Currently, 202 of 276 patients have completed at least 24 months of follow-up. In eight of 276 patients, groin recurrences were observed after a negative sentinel node procedure (two with multifocal disease). The actuarial groin recurrence rate after 2 years was 3\% (95\% CI, 1\% to 6\%) for all patients and $2.3 \%$ (95\% CI, $1.0 \%$ to $5.0 \%)$ for patients with unifocal disease. Median time to groin recurrence was 12 months (range, 5 to 16 months; Fig 2A). All patients with a groin recurrence underwent a bilateral inguinofemoral lymphadenectomy and adjuvant (chemo)radiation. Locoregional control was achieved in four of eight patients. Six patients died of disease, whereas two are alive without evidence of disease ( 50 and 6 months after treatment of recurrence). Two patients had only one sentinel node removed, whereas the lymphoscintigram showed two sentinel nodes. Two patients had micrometastases at ultrastaging that were only detected at pathology review. In the remaining four patients, no obvious explanation for failure was found, but two of the patients had multifocal disease.

Local recurrences and disease-specific and overall survival. During follow-up, $16(5.8 \%)$ of 276 patients died of intercurrent disease, and 10 patients $(3.6 \%)$ died of vulvar cancer. In 34 patients (12.3\%), a local recurrence occurred (median time to recurrence, 16 months; range, 2 to 67 months). Treatment in these patients was at the discretion of the individual center. Five patients with local recurrence eventually died of distant metastases $(15,18,22,41$, and 41 months after primary treatment). Figure $2 \mathrm{~B}$ shows the disease-specific survival curve for patients with negative sentinel nodes. The 3-year disease-specific survival rate for patients with unifocal vulvar disease and negative sentinel nodes was $97.0 \%$.

\section{DISCUSSION}

This prospective study is the largest study on surgical techniques ever performed in vulvar cancer. The low groin recurrence rate $(3 \%$ including multifocal disease; $2.3 \%$ in unifocal vulvar disease) and excellent disease-specific survival rate of $97 \%$ at 3 years in sentinel nodenegative patients suggest that the sentinel node procedure is a safe alternative to elective inguinofemoral lymphadenectomy for selected vulvar cancer patients. Apart from two small, single-institution series from which no conclusions with respect to efficacy and safety could be

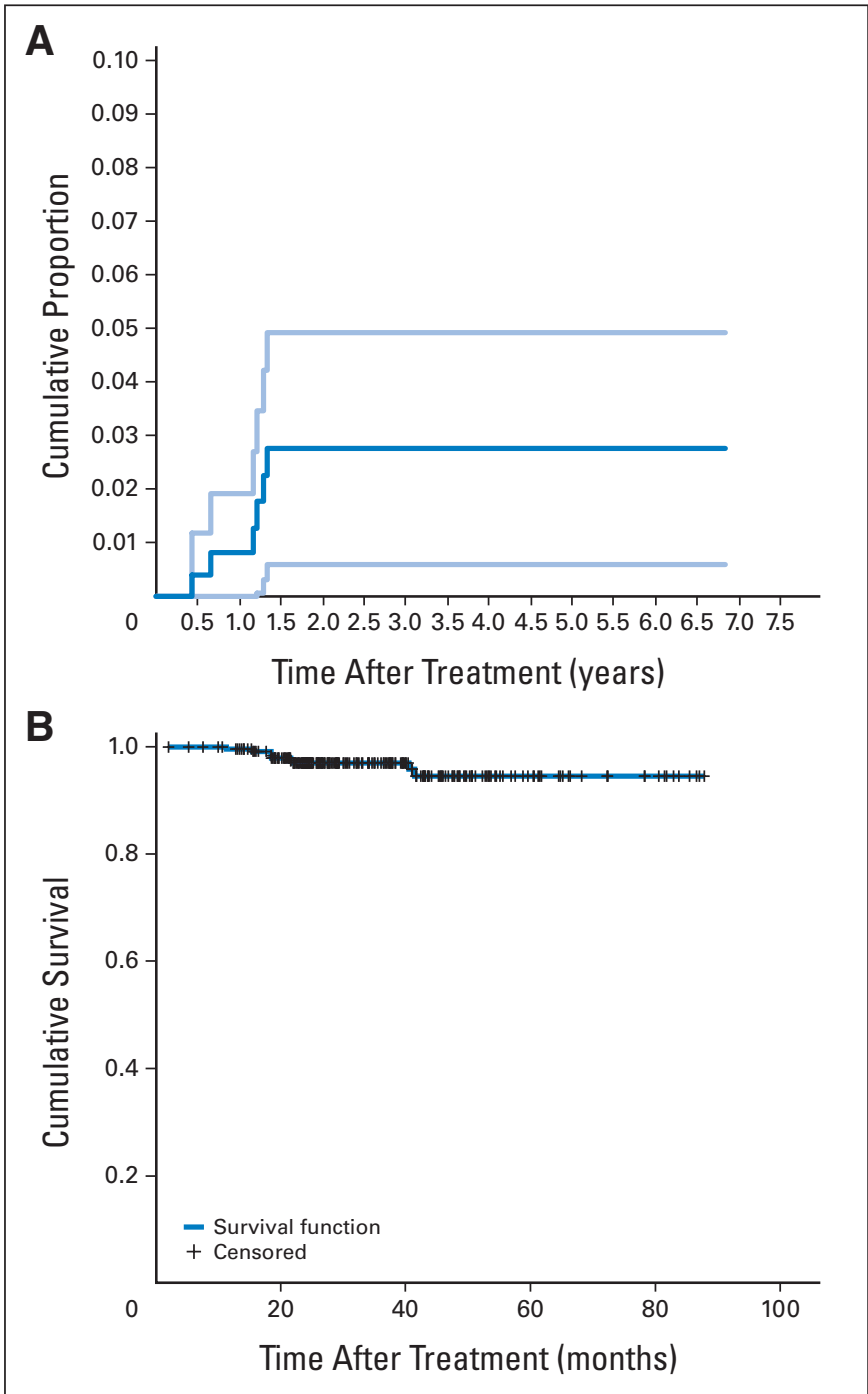

Fig 2. (A) Cumulative proportion of groin recurrences in patients with unifocal vulvar cancer and negative sentinel node (dark blue line); $95 \% \mathrm{Cls}$ are also given (light blue lines). (B) Disease-specific survival curve for early-stage, unifocal vulvar cancer patients with a negative sentinel node.

drawn, comparable data in vulvar cancer do not exist. ${ }^{32,33}$ The primary end point in our study was groin recurrence rate because groin recurrences are often fatal $^{9}$ and of major concern when considering a less radical approach of the groin in vulvar cancer. Groin recurrence rates in often small, retrospective studies vary from $0 \%$ to $5.8 \%$ for lymph node-negative patients ${ }^{8,10,11}$ (see Appendix). The groin recurrence rate in sentinel node-negative patients in the current study seems to be at least comparable to that reported for patients with early-stage vulvar cancer treated with formal lymphadenectomy of any type.

The nodal recurrence risk may seem less favorable when compared with that seen after the sentinel node procedure in the treatment of breast cancer $(0.1 \%$ to $0.3 \%){ }^{35}$ This might be partly explained by the fact that the majority of breast cancer patients will receive adjuvant treatment after surgery, whereas vulvar cancer patients with a negative sentinel node do not receive any adjuvant treatment. However, from studies where the sentinel node procedure was followed by formal lymphadenectomy, the variation in false-negative rates (range, $0 \%$ to 
$27 \%$; average, $6.7 \%)^{17}$ seems to be higher in breast cancer than the variation found for the sentinel node procedure in vulvar cancer (typically $0 \%$ to $2 \%$ ).

The excellent performance of the sentinel node technique in vulvar cancer is perhaps surprising given that the accuracy of the sentinel node procedure is strongly associated with the experience of the surgeon. In the Axillary Lymphatic Mapping Against Nodal Axillary Clearance trial in breast cancer, centers were only allowed to participate after at least 40 successful sentinel node procedures followed by full axillary lymphadenectomy. ${ }^{21}$ Even in countries where treatment of vulvar cancer is centralized, such figures are not realistic because of the rarity of the disease. It may be that the more superficial location of the inguinofemoral region compared with the axillary region facilitates easier and thus more accurate detection, even by less experienced surgeons such as in our multicenter study. However, in depth analysis of our eight patients with false-negative results revealed surgeon- and procedure-related factors in four of eight patients, stressing the necessity of strict adherence to the sentinel node protocol by the entire multidisciplinary team to prevent false-negative results. Therefore, implementation of the sentinel node procedure in routine treatment of early-stage vulvar cancer requires quality control at each step of this multidisciplinary procedure, including injection of radioactive tracer by either the surgeon or a nuclear medicine physician familiar with vulvar anatomy, careful interpretation of lymphoscintigram, a surgeon with successful experience (sentinel node procedure followed by full lymphadenectomy) in at least 10 patients, and a pathology department experienced in ultrastaging of the sentinel nodes. All sentinel nodes that are negative at routine HE examination need to be further analyzed by ultrastaging. Finally, to keep the experience at a high level, an exposure of at least five to 10 patients per year per surgeon should be regarded as a minimum figure. In a rare tumor such as vulvar cancer, this will require centralization of early-stage vulvar cancer treatment in oncology centers.

Two years after the activation of the study, a protocol amendment was made when two groin recurrences occurred in 19 patients with multifocal disease. Analysis revealed that peritumoral injection of the tracer in multifocal disease seemed likely to not be representative of the extent of the tumors. Fortunately, no further recurrences were observed in the additional 19 patients with negative sentinel nodes already registered on the study. If all patients with multifocal disease are excluded, only six groin recurrences occurred, pointing to an even lower groin recurrence rate (six of 259 patients; $2.3 \%$; $95 \% \mathrm{CI}, 1 \%$ to $5 \%)$. Apart from multifocal disease, we also excluded patients with tumors greater than $4 \mathrm{~cm}$ from our study. Although this figure was arbitrarily chosen, representative injection of tracer around larger tumors is less likely. Furthermore, nodal involvement increases with lesion size, and alterations in lymphatic flow that may occur with large involved nodes could further increase the likelihood of false-negative results. ${ }^{35}$
The main purpose of the introduction of the sentinel node technique in early-stage vulvar cancer is reduction of treatment-related morbidity in patients without metastatic nodes. We have shown that sentinel node removal alone resulted in less short- and long-term morbidity compared with inguinofemoral lymphadenectomy. The rates of short- and long-term morbidity after inguinofemoral lymphadenectomy in this study were comparable to those reported in the literature. ${ }^{10,12-14}$ Long-term morbidity was especially high in patients who underwent inguinofemoral lymphadenectomy followed by radiotherapy. In the ongoing Groningen International Study on Sentinel Nodes in Vulvar Cancer-II study, patients with a metastatic sentinel node will receive radiotherapy instead of inguinofemoral lymphadenectomy to reduce morbidity caused by double-modality treatment.

In conclusion, our study demonstrates that the introduction of the sentinel node procedure in the management of early-stage vulvar cancer performed by a quality-controlled multidisciplinary team results in decreased morbidity without compromising groin recurrence or survival rates. Sentinel node detection should be discussed as a safe treatment option when counseling a patient with unifocal, early-stage vulvar cancer.

\section{AUTHORS' DISCLOSURES OF POTENTIAL CONFLICTS OF INTEREST}

The author(s) indicated no potential conflicts of interest.

\section{AUTHOR CONTRIBUTIONS}

Conception and design: Ate G.J. Van der Zee, Joanne A. De Hullu, Anca C. Ansink, Ignace Vergote, René H. Verheijen, Jacobus Van der Velden, Administrative support: Ate G.J. Van der Zee

Provision of study materials or patients: Ate G.J. Van der Zee, Maaike H. Oonk, Joanne A. De Hullu, Anca C. Ansink, Ignace Vergote, René H. Verheijen, Angelo Maggioni, Katja N. Gaarenstroom, Peter J. Baldwin, Eleonora B. Van Dorst, Jacobus Van der Velden, Ralph H. Hermans, Hans van der Putten, Pierre Drouin, Achim Schneider

Collection and assembly of data: Ate G.J. Van der Zee, Maaike H. Oonk, Joanne A. De Hullu, Anca C. Ansink, Ignace Vergote, René H. Verheijen, Angelo Maggioni, Katja N. Gaarenstroom, Peter J. Baldwin, Eleonora B. Van Dorst, Jacobus Van der Velden, Ralph H. Hermans, Hans van der Putten, Pierre Drouin, Achim Schneider

Data analysis and interpretation: Ate G.J. Van der Zee, Maaike H. Oonk, Wim J. Sluiter

Manuscript writing: Ate G.J. Van der Zee, Maaike H. Oonk, Joanne A. De Hullu, Anca C. Ansink, Ignace Vergote, Peter J. Baldwin, Jacobus Van der Velden, Wim J. Sluiter

Final approval of manuscript: Ate G.J. Van der Zee, Maaike H. Oonk, Joanne A. De Hullu, Anca C. Ansink, Ignace Vergote, René H. Verheijen, Angelo Maggioni, Katja N. Gaarenstroom, Peter J. Baldwin, Eleonora B. Van Dorst, Jacobus Van der Velden, Ralph H. Hermans, Hans van der Putten, Pierre Drouin, Achim Schneider, Wim J. Sluiter

\section{REFERENCES}

1. Booth $H$, Cooper N, Quinn M (eds): Cance statistics registration: Registration of cancer diagnosed in 2000, England. http://www.statistics.gov.uk/ downloads/theme_health/Mb1_31/Mb1_31.pdf

2. US Cancer Statistics Working Group.United States Cancer Statistics: 2003 Incidence and Mortality. Atlanta, GA, US Department of Health and Human Services, Centers for Disease Control and Prevention, and National Cancer Institute, 2006

3. Hemminki K, Li X, Vaittinen P: Time trends in the incidence of cervical and other genital squamous cell carcinomas and adenocarcinomas in Sweden, 1958-1996. Eur J Obstet Gynecol Reprod Biol 101: 64-69, 2002

4. Homesley HD, Bundy BN, Sedlis A, et al: Assessment of current International Federation of Gynecology and Obstetrics staging of vulvar carcinoma relative to prognostic factors for survival: A Gynecologic Oncology Group study. Am J Obstet Gynecol 164:997-1004, 1991

5. Burger MP, Hollema $H$, Emanuels $A G$, et al: The importance of the groin node status for the survival of T1 and T2 vulval carcinoma patients. Gynecol Oncol 57:327-334, 1995

6. Hacker NF, Leuchter RS, Berek JS, et al: Radical vulvectomy and bilateral inguinal lymphadenectomy through separate groin incisions. Obstet Gynecol 58:574-579, 1981 
7. De Hullu JA, Hollema H, Lolkema $S$, et al: Vulvar carcinoma: The price of less radical surgery. Cancer 95:2331-2338, 2002

8. Bell JG, Lea JS, Reid GC: Complete groin lymphadenectomy with preservation of the fascia lata in the treatment of vulvar carcinoma. Gynecol Oncol 77:314-318, 2000

9. Katz A, Eifel PJ, Jhingran A, et al: The role of radiation therapy in preventing regional recurrences of invasive squamous cell carcinoma of the vulva. Int J Radiat Oncol Biol Phys 57:409-418, 2003

10. Stehman FB, Bundy BN, Dvoretsky PM, et al: Early stage I carcinoma of the vulva treated with ipsilateral superficial inguinal lymphadenectomy and modified radical hemivulvectomy: A prospective study of the Gynecologic Oncology Group. Obstet Gynecol 79:490-497, 1992

11. Kirby TO, Rocconi RP, Numnum TM, et al: Outcomes of stage I/II vulvar cancer patients after negative superficial inguinal lymphadenectomy. Gynecol Oncol 98:309-312, 2005

12. De Hullu JA, Ansink AC, Tymstra $T$, et al: What doctors and patients think about falsenegative sentinel lymph nodes in vulvar cancer. J Psychosom Obstet Gynaecol 22:199-203, 2001

13. Rouzier R, Haddad B, Dubernard G, et al: Inguinofemoral dissection for carcinoma of the vulva: Effect of modifications of extent and technique on morbidity and survival. J Am Coll Surg 196:442450, 2003

14. Gaarenstroom KN, Kenter GG, Trimbos JB, et al: Postoperative complications after vulvectomy and inguinofemoral lymphadenectomy using separate groin incisions. Int J Gynecol Cancer 13:522527, 2003

15. Oonk MH, Hollema $H$, de Hullu JA, et al: Prediction of lymph node metastases in vulvar cancer: A review. Int J Gynecol Cancer 16:963-971, 2006

16. Selman TJ, Luesley DM, Acheson $N$, et al: A systematic review of the accuracy of diagnostic tests for inguinal lymph node status in vulvar cancer. Gynecol Oncol 99:206-214, 2005

17. Kim $T$, Giuliano $A E$, Lyman GH: Lymphatic mapping and sentinel lymph node biopsy in earlystage breast carcinoma: A metaanalysis. Cancer 106:4-16, 2006

18. Thompson JF, Shaw HM: Sentinel node mapping for melanoma: Results of trials and current applications. Surg Oncol Clin N Am 16:35-54, 2007

19. Purushotham $A D$, Upponi $S$, Klevesath $M B$, et al: Morbidity after sentinel lymph node biopsy in primary breast cancer: Results from a randomized controlled trial. J Clin Oncol 23:4312-4321, 2005

20. Veronesi U, Paganelli G, Viale G, et al: Sentinel-lymph-node biopsy as a staging procedure in breast cancer: Update of a randomised controlled study. Lancet Oncol 7:883-890, 2006

21. Mansel RE, Fallowfield $L$, Kissin $M$, et al: Randomized multicenter trial of sentinel node biopsy versus standard axillary treatment in operable breast cancer: The ALMANAC trial. J Natl Cancer Inst 98:599-609, 2006

22. Wilke LG, McCall LM, Posther KE, et al: Surgical complications associated with sentinel lymph node biopsy: Results from a prospective international cooperative group trial. Ann Surg Oncol 13:491-500, 2006

23. Lucci A, McCall LM, Beitsch PD, et al: Surgical complications associated with sentinel lymph node dissection (SLND) plus axillary lymph node dissection compared with SLND alone in the American College of Surgeons Oncology Group Trial Z0011. J Clin Oncol 25:3657-3663, 2007

24. Ansink AC, Sie-Go DMDS, van der Velden J, et al: Identification of sentinel lymph nodes in vulvar carcinoma patients with the aid of a patent blue $\mathrm{V}$ injection. Cancer 86:652-656, 1999

25. De Cicco $C$, Sideri M, Bartolomei $M$, et al: Sentinel node biopsy in early vulvar cancer. $\mathrm{Br} J$ Cancer 82:295-299, 2000
26. De Hullu JA, Hollema $H$, Piers DA, et al: Sentinel lymph node procedure is highly accurate in squamous cell carcinoma of the vulva. J Clin Oncol 18:2811-2816, 2000

27. Levenback C, Coleman RL, Burke TW, et al: Intraoperative lymphatic mapping and sentinel node identification with blue dye in patients with vulvar cancer. Gynecol Oncol 83:276-281, 2001

28. Sliutz G, Reinthaller A, Lantzsch $T$, et al: Lymphatic mapping of sentinel nodes in early vulvar cancer. Gynecol Oncol 84:449-452, 2002

29. Moore RG, DePasquale SE, Steinhoff MM, et al: Sentinel node identification and the ability to detect metastatic tumor to inguinal lymph nodes in squamous cell cancer of the vulva. Gynecol Oncol 89:475-479, 2003

30. Puig-Tintoré LM, Ordi J, Vidal-Sicart $S$, et al: Further data on the usefulness of sentinel lymph node identification and ultrastaging in vulvar squamous cell carcinoma. Gynecol Oncol 88:29-34, 2003

31. De Hullu JA, Doting E, Piers DA, et al: Sentinel lymph node identification with technetium-99mlabeled nanocolloid in squamous cell cancer of the vulva. J Nucl Med 39:1381-1385, 1998

32. Vidal-Sicart S, Puig-Tintoré LM, Lejárcegui JA, et al: Validation and application of the sentinel lymph node concept in malignant vulvar tumours. Eur J Nucl Med Mol Imaging 34:384-391, 2007

33. Nyberg $\mathrm{RH}$, livonen $\mathrm{M}$, Parkkinen $\mathrm{J}$, et al: Sentinel node and vulvar cancer: A series of 47 patients. Acta Obstet Gynecol Scand 86:615-619, 2007

34. Terada KY, Shimizu DM, Jiang CS, et al: Outcomes for patients with T1 squamous cell cancer of the vulva undergoing sentinel node biopsy. Gynecol Oncol 102:200-203, 2006

35. de Hullu JA, Oonk MH, Ansink AC, et al: Pitfalls in the sentinel lymph node procedure in vulvar cancer. Gynecol Oncol 94:10-15, 2004

\section{Appendix}

The Appendix is included in the full-text version of this article, available online at www.jco.org. It is not included in the PDF version (via Adobe ${ }^{\circledR}$ Reader $^{\circledR}$ ). 